\title{
The Way to Control the Flux in Sulfuric Acid Producing
}

\author{
Rui Cai \\ Computer And Automation Institute \\ Tianjin Polytechnic University \\ Tianjin 300160 China \\ E-mail:cairui715@126.com \\ Ping Wang \\ Computer And Automation Institute \\ Tianjin Polytechnic University \\ Tianjin 300160 China \\ E-mail:wangping@gmail.com
}

\begin{abstract}
A flow ratio control system using is introduced. It is composed of Sensor, Flow meter and Mitsubishi PLC, which is the core of the control system, and some other equipment as an example that Potassium Chloride and Sulfuric acid make Potassium Sulfate to introduce this control system using in the chemical industry. This control system controls the flowing of the Sulfuric acid based on the flowing of Potassium Chloride, satisfying the demanded of the technological requirement. The result of practiced application indicates that this system has high control precision and good Stability.
\end{abstract}

Keywords: Frequency-varying timing, Flow control, PLC

\section{Introduction}

In the process of producing, the system of mixing two or more materiel with a certain ratio is called as Proportional Control system. In the chemical industry, the controlling of flux is very important. This text introduced a system of flux Proportional Controlling. After the experiment and practice, the system is proved that it is provided with several merit such as more simple structure, less error of stability and higher precision of controlling.

\section{Theory}

The Proportional Control systems are composed by open loop Proportional Control systems, single Closed-loop Proportional Control system and double Closed-loop Proportional Control system. It is the most simple that open loop controlling system. The single Closed-loop Proportional Control system is used for conquering the flaw of open loop Proportional Control system. But it also owns its shortage that the main flux is not controlled as a Closed-loop. The system in the text is used the double Closed-loop Proportional Control system.

The first Closed-loop controlling system is the flux Closed-loop controlling system of the Potassium Chloride itself (fig 1). After setting, with the effect of Closed-loop modulation, the system can eliminate the effect of interfere, and make the flux of Potassium Chloride stabile at the number of setting. The main flux Closed-loop controlling system is a constant value one.

The second one is subsidiary flux Sulfuric acid Closed-loop controlling system, which input is the product which equal the flux signal $\mathrm{Q}_{1}$ of Potassium Chloride multiplied ratio coefficient $\mathrm{K}_{1}$. The system is composed by subsidiary controller 1, Sulfuric acid pump inverter, Sulfuric acid pump, survey point 3 and inverter 2. It is a servo system.

\section{System designing of flow Proportional Control}

\section{1 structure}

The flux of Potassium Chloride and Sulfuric acid Proportional Control system is composed by Mitsubishi FX ${ }_{2 \mathrm{NC}}$ PLC, pump which is able to bear to be corrupt, inverter of Siemens MM440, screw of computing and electromagnetic flowmeter. This problem has been studied previously (liao, changchu, 2006) The system is showed as fig2. .

\subsubsection{Mitsubishi $\mathrm{FX}_{2 \mathrm{NC}}$ PLC}

Mitsubishi $\mathrm{FX}_{2 \mathrm{NC}} \mathrm{PLC}$ is provided with higher ratio of performance and volume, and better communication function. It can be fixed in smaller place than normal PLC. Its linker as I/O type may reduce the cost of linking and saving hours. The number of I/O can reach 256. It can link 4 special function modules at most. 


\subsubsection{Uncorrupted pump}

Sulfuric acid is a kind of medium which can corrupt others, so the pump for transportation must use uncorrupted pump. The system use fluoric plastic off-center pump typed IHF 65 50-160, whose inlet diameter is $65 \mathrm{~mm}$, whose exit diameter is $50 \mathrm{~mm}$, whose impeller normal diameter is $160 \mathrm{~mm}$, whose rotate speed is $2900 \mathrm{r} / \mathrm{min}$, whose flux is $25 \mathrm{~m}^{3} / \mathrm{h}$, whose lifting height is $32 \mathrm{~m}$ and whose motor power is $5.5 \mathrm{kw}$. It use timing of frequency conversion. It should use special frequency conversion for water pump towards the equipment of pump. The motor power of fluoric plastic off-center pump typed IHF $6550-160$ is $5.5 \mathrm{kw}$, which choose special inverter of Siemens MM440 for water pump, which owns A level filter insides.

\subsubsection{Inverter of Siemens MM440}

The flow velocity of the substance is computed by the output impulse from the motor coder. It may effect the normal work of computing screw. As a result, it is vector controlling inverter of Siemens MM440 that is used in the system whose power supply is $380 \mathrm{VAC}$, whose rating output power is $5.5 \mathrm{KW}$, whose rating output current is $13.2 \mathrm{~A}$. In order to make the rotate speed of screw axial stabile and to compute the flow velocity of the substance, it takes the vector controlling with velocity coder. The inverter needs to add impulse coder impulse managing module (coder module for short) and coder.

\subsection{4 computing screw}

Potassium Chloride is a kind of materiel like powder. The screw of computing is used to transport the powder materiel, to measure at once and to control the flow. Computing screw of LSC type of wanxide company is used in the system.

After potassium Chloride powder comes into the powder storehouse, it goes into screw transportation, and then it moves to output door. The sensor under the output door will transport the weight signal to analog signal input module of PLC. At the same tine, coder at the end of non-loading axis measures the number of round and the velocity. It makes the output impulse transported to the high-speed counters part of PLC. After the computing of CPU, the value of flow and accumulation weight of Potassium Chloride. The controlling signal from analog signal output module controls inverter and frequency conversion motor in order to control the flow of Potassium Chloride which is the initiative value of the system. The flow of powder is showed as:

$$
Q=K \cdot q \cdot V
$$

Where: Q - the flow of the computing screw, $\mathrm{kg} / \mathrm{s} ; \mathrm{K}$ - the coefficient of weight; q-linear load of powder in the screw, $\mathrm{kg} / \mathrm{m} ; \mathrm{V}$ - the flow velocity of powder in the screw, $\mathrm{m} / \mathrm{s}$.

The powder moves along the spiraled track in the screw, so the flow velocity is not easy to be measured. It is usually converted by coder which measures the velocity of motor. The computing screw is showed as fig3.

\subsubsection{Electromagnetic flowmeter}

Electromagnetic flowmeter is mostly used to measured the volume flow of electric liquid and liquid-solid. The result is independent of the temperature, mucosity, density, pressure and the component of liquid and solid, and also not affected when conductance changes in a larger range. So, after normal water demarcating, the electromagnetic flowmeter which is a real volume flowmeter is used to measure the flow of other electric liquid or liquid-solid. It takes WSDQ type electromagnetic flowmeter made in wanshan automatization instrument Ltd in Guangdong province.

\section{2 the design of PLC controlling system}

The output signal of weighting Potassium Chloride device and Sulfuric acid electromagnetism flowmeter are both 4 20mA signal which is sent to the current inputland input2 of analog output module FX2N-4AD. It owns 4 12-bit analog input channels which may current signal $(4 \sim 20 \mathrm{~mA})$ or voltage signal $(-10 \sim 10 \mathrm{~V})$. The conversion velocity is $15 \mathrm{~ms} /$ channel or $6 \mathrm{~ms} /$ channel (high speed).

The computing screw and Sulfuric acid pump are with frequency conversion timing system. The controlling signals of the two inverter come from analog output module FX2N-4DA of PLC system. It owns 12 4-bit channels, whose output may current signal $(4 \sim 20 \mathrm{~mA})$ or voltage signal $(-10 \sim 10 \mathrm{~V})$. The conversion velocity equal to the value that four channels $2.1 \mathrm{~ms}$ take $8 \mathrm{I} / \mathrm{O}$ pin. This problem has been studied previously (cai, erfu and chen, shuhui, 2004).

\subsection{1 intercalating parameter data memorizer address}

The value of intercalating computing screw input which equal to the flow of Potassium Chloride: D1D0, 8-bit BCD code.

The flow ratio coefficient of Potassium Chloride and Sulfuric acid: D3D2/D9D8.

The memorizer address of Potassium Chloride flow in fact: M500, 16-bit binary code.

The memorizer address of Sulfuric acid flow in fact: M501, 16-bit binary code. 
The memorizer address of 16-step gain: from the first step to the tenth step: D110 D119,D140 D149.

\subsection{2 accommodating inverter}

when MM430 inverter is used to control the loadings such as pumps, the starting frequency must be given attention to in order to producing over modulation and shocking. After the sizes of pipelines and pump are confirmed, the flow starting frequency is also confirmed. When the frequency is more than $19 \mathrm{~Hz}$, the flow output is not null. So before measuring, the open loop experiment must be taken. The controlling arithmetic is restricted by measured first frequency. In the first frequency, taking open-loop module, the output signal is slope function (linearity gain voltage signal). After the first frequency produces flow, it changes to closed-loop controlling module, which is composed with proportion controlling module and proportion-integral controlling module. At first, the proportion controlling module runs. And then, it turns to the proportion-integral controlling module when the flow is nearly the enactment value in fact. In two modules controlling, the system dispels the large fluctuating of flow, improves the flow following capability, and also dispels the stability error.

\subsubsection{PID controlling}

The controlling instruction of $\mathrm{FX}_{2 \mathrm{NC}}$ type:

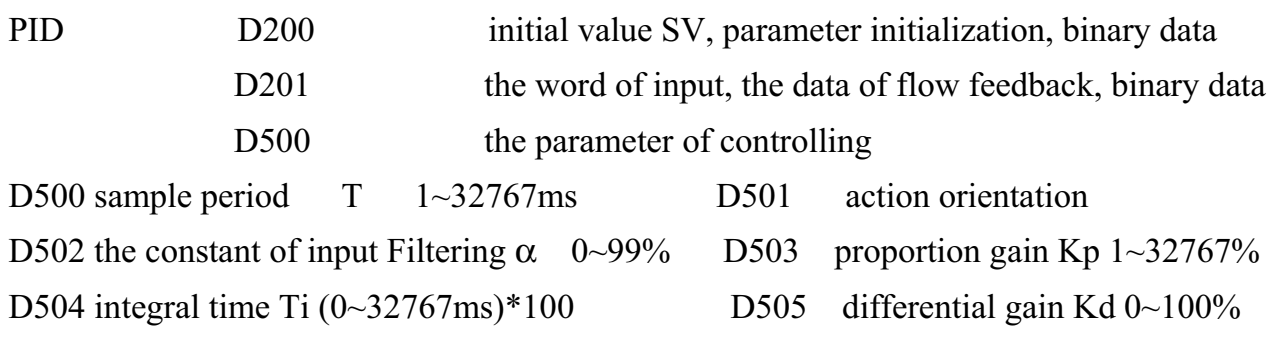

D506 differential time $\mathrm{Td}(0 \sim 32767) * 100 \mathrm{~ms}$

\subsection{4 the modulation of system}

(1) D503 (proportion gain $\mathrm{Kp}$ ): about $8000(\mathrm{BCD}$ code), the less value of $\mathrm{P}$, the more overshoot, the more times of oscillatory.

(2) D504 (integral time Ti) according to error data (D50), Ti is measured off by 10 steps, which is transferred automatically when the system runs in order to restrain overshoot and enhance the velocity of response. When the given value becomes bigger abruptly, the biggest overshoot is less $5 \%$.

\section{4. conclusion}

The proportion controlling system or proportion-integral controlling system which is gain value and integral time constant are settled parameter, so it is hard to control the following performance and anti-interfere performance and to arrive the aim of restraining over modulation and enhancing the velocity of response. In the system, according to the value of flow closed-loop controlling system error, the proportion coefficient and integral time constant are divided by 10 steps which is automatically transfer appropriately when the system runs. The system owns the capability of restraining overshoot and enhancing the velocity of response. So the system may take better application effect.

\section{References}

Cai, Erfu, \& Chen, Shuhui (2004). The System Designing in Chemical Factory. Chemical Machine Press. Liao, Changchu (2006). The Programming and Application of PLC of FX. China Machine Press. 


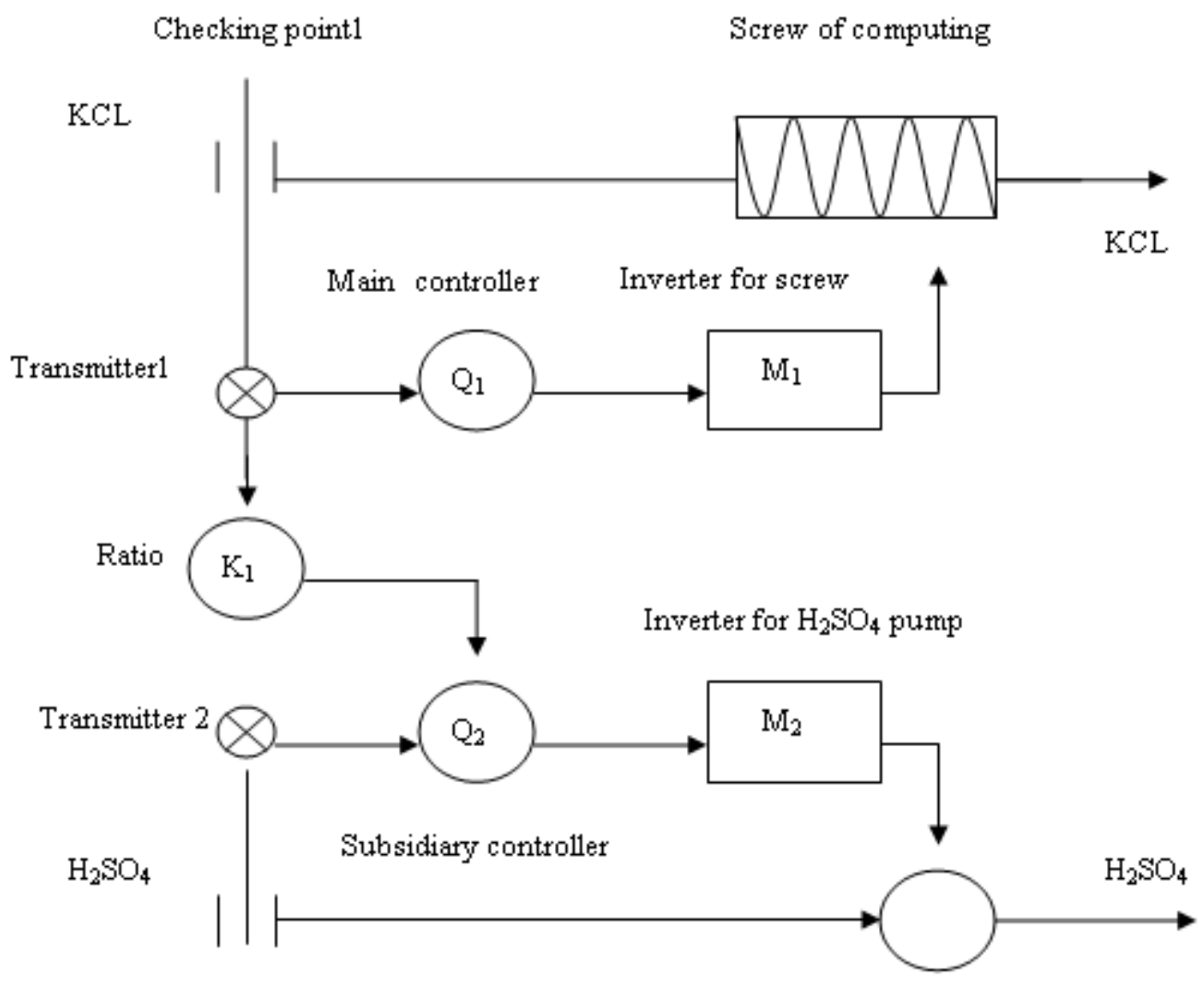

Figure 1. KCL- $\mathrm{H}_{2} \mathrm{SO}_{4}$ double closed-loop flow Proportional Control system

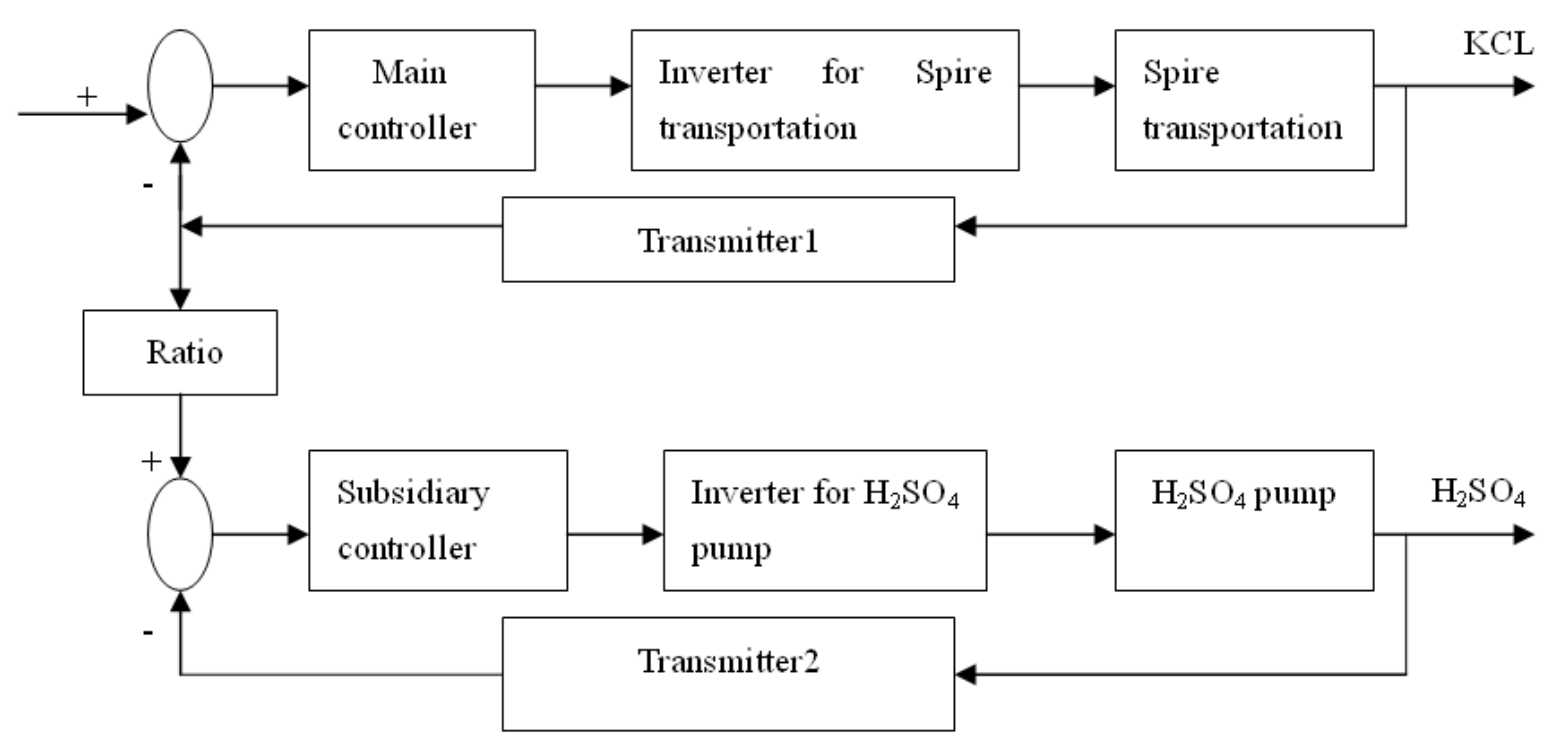

Figure 2. flow Proportional Control system 


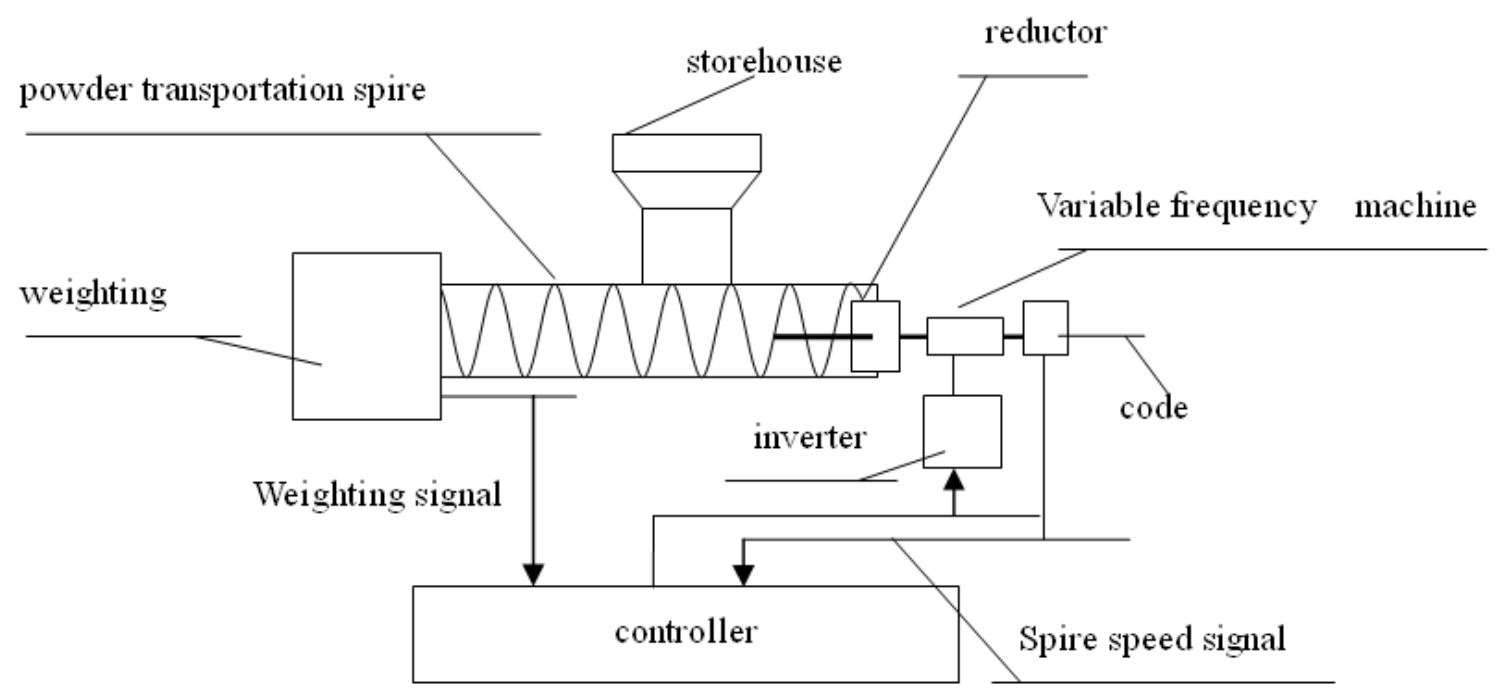

Figure 3. measure spire sketch map 